\title{
Four New Ursane-Type Triterpenes, Olibanumols K, L, M, and N, from Traditional Egyptian Medicine Olibanum, the Gum-Resin of Boswellia carterii
}

\author{
Toshio Morikawa, ${ }^{a, b}$ Hideo Oominami, ${ }^{a}$ Hisashi Matsuda,${ }^{a}$ and Masayuki Yoshikawa $*, a$ \\ ${ }^{a}$ Kyoto Pharmaceutical University; Misasagi, Yamashina-ku, Kyoto 607-8412, Japan: and ${ }^{b}$ Pharmaceutical Research and \\ Technology Institute, Kinki University; 3-4-1 Kowakae, Higashi-Osaka, Osaka 577-8502, Japan. \\ Received July 6, 2010; accepted August 6, 2010; published online August 6, 2010
}

Four new ursane-type triterpenes, olibanumols K (1), L (2), M (3), and N (4), were isolated from traditional Egyptian medicine olibanum, the exuded gum-resin from Boswellia carterii BIRDw. Their structures were elucidated on the basis of chemical and physicochemical evidence.

Key words olibanumol; olibanum; Boswellia carterii; triterpene; Burseraceae

During the course of our studies on bioactive constituents from Egyptian and Yemeni natural medicines, ${ }^{1-12)}$ we found that $80 \%$ aqueous acetone extract of exuded gum-resin of Boswellia carterii, so called olibanum (frankincense), showed anti-inflammatory effect by monitoring the inhibitory activity of nitric oxide (NO) production induced by lipopolysaccharide (LPS) in mouse peritoneal macrophages. ${ }^{10)}$ From the extract, three monoterpenes, olibanumols $\mathrm{A}-\mathrm{C}$, a diterpene, olibanumol D, and six triterpenes, olibanumols $\mathrm{E}$ - J w were isolated together with five monoterpenes and two triterpenes, epilupeol (5) and isofouquierol (21). ${ }^{10,13)}$ Among them, several constituents including olibanumols $\mathrm{A}, \mathrm{D}, \mathrm{E}$, and $\mathrm{H}$ exhibited the inhibitory effect on the NO production. ${ }^{10)}$ As a continuing study on this natural medicine, we further isolated four new ursane-type triterpenes, olibanumols K (1), L (2), M (3), and N (4), together with 19 known triterpenes. This paper deals with the isolation and structure elucidation of these newly isolated triterpenes $(\mathbf{1}-\mathbf{4}) .^{14)}$

The gum-resin from $B$. carterii collected in Yemen and purchased at Cairo of Egypt was extracted with $80 \%$ aqueous acetone at room temperature to give an aqueous acetone extract (78.9\% from the natural medicine), as was reported. ${ }^{10)}$ By the intensive chromatographies on the aqueous acetone extract, $1(0.0013 \%), 2(0.043 \%), 3(0.0043 \%)$, and 4 $(0.0035 \%)$ have been newly isolated together with seven lupane-type triterpenes, epilupeol acetate $^{15)}(6,2.87 \%)$, lup-20(30)-ene-3 $\alpha, 29-$ diol $^{16)}(7,0.0050 \%)$, glochidiol ${ }^{17)}(\mathbf{8}$ $0.0017 \%)$, lupeol $^{18)}(\mathbf{9}, 0.72 \%)$, lup-20(29)-ene- $\alpha, 3 \beta$-diol ${ }^{19)}$ (10, 0.0026\%), 3 $\beta$-acetoxylup-20(29)-en-11 $\beta$-ol ${ }^{20)}(\mathbf{1 1}, 0.043$ $\%)$, and lupenone ${ }^{21,22)}(\mathbf{1 2}, 0.33 \%)$, five ursane-type triterpenes, urs-9(11),12-dien-3 $\beta$-ol ${ }^{23,24)}(\mathbf{1 3}, 0.0012 \%)$, neoilexonol $^{25)}(\mathbf{1 4}, 0.039 \%)$, neoilexonol acetate 26,27$)(15,0.27 \%)$, urs-12-ene-3 $\beta, 11 \alpha$-diol ${ }^{28)}(\mathbf{1 6}, 0.0041 \%)$, and urs-12-ene$3 \alpha, 11 \alpha-\operatorname{diol}^{28)}(\mathbf{1 7}, 0.015 \%)$, and five dammarane-type and two nordammarane-type triterpenes, dammarenediol $\mathrm{II}^{29,30)}$ (18, 0.089\%), dammarenediol II acetate ${ }^{30)}$ (19, 0.45\%), 3-Oacetyl-3 $\beta, 20 S, 24$-trihydroxydammar-25-ene ${ }^{31)}(\mathbf{2 0}, 0.0022 \%)$, isofouquierol acetate ${ }^{31,32)}(22,0.066 \%)$, ocotillol acetate ${ }^{33)}$ (23, $0.0044 \%), 3 \beta$-hydroxymansumbin-13(17)-en-16-one ${ }^{34}$ $(\mathbf{2 4}, 0.0065 \%)$, and mansumbinol ${ }^{35)}(\mathbf{2 5}, 0.0071 \%)$.

Structure of Olibanumol K (1) Olibanumol K (1) was obtained as a white powder with positive optical rotation $\left([\alpha]_{\mathrm{D}}^{27}+16.2\right.$ in $\left.\mathrm{MeOH}\right)$. The IR spectrum of $\mathbf{1}$ showed absorption bands at $1725 \mathrm{~cm}^{-1}$ ascribable to an ester car-
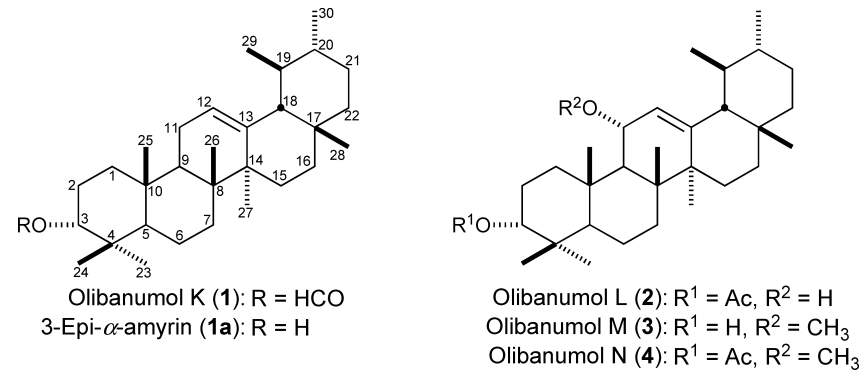

Chart 1

bonyl function. In the positive-ion fast atom bombardment (FAB)-MS of 1, a quasimolecular ion peak was observed at $m / z 477(\mathrm{M}+\mathrm{Na})^{+}$, and high-resolution positive-ion FABMS analysis revealed the molecular formula of $\mathbf{1}$ to be $\mathrm{C}_{31} \mathrm{H}_{50} \mathrm{O}_{2}$. The ${ }^{1} \mathrm{H}-\left(\mathrm{CDCl}_{3}\right)$ and ${ }^{13} \mathrm{C}-\mathrm{NMR}$ (Table 1) spectra of 1 , which were assigned by various NMR experiments, ${ }^{36}$ ) showed signals assignable to eight methyls $[\delta 0.80,0.89$, $0.92,0.92,1.11,1.13$ (3H each, all s, 28, 23, 24, 25, 27, 26$\left.\mathrm{H}_{3}\right), 0.90\left(3 \mathrm{H}, \mathrm{d}, J=7.4 \mathrm{~Hz}, 29-\mathrm{H}_{3}\right), 0.92(3 \mathrm{H}, \mathrm{d}, J=7.0 \mathrm{~Hz}$, $\left.30-\mathrm{H}_{3}\right)$, a methine bearing an oxygen function $[\delta 4.77(1 \mathrm{H}$, brs, 3-H)], an olefin $[\delta 5.13(1 \mathrm{H}$, dd-like, 12-H)], and a formyl group $[\delta 8.13(1 \mathrm{H}, \mathrm{s}, \underline{\mathrm{HCO}-})]$ together with nine methylenes, five methines, and six quaternary carbons. The ursane-type triterpene structure of $\mathbf{1}$ was constructed on the basis of the ${ }^{1} \mathrm{H}-{ }^{1} \mathrm{H}$ correlation spectroscopy $\left({ }^{1} \mathrm{H}-{ }^{1} \mathrm{H}\right.$ COSY $)$ and heteronuclear multiple bond correlation (HMBC) experiments as shown in Fig. 1. Thus, ${ }^{1} \mathrm{H}-{ }^{1} \mathrm{H}$ COSY experiment indicated the presence of partial structures in bold lines. In the HMBC experiment on 1, long-range correlations were observed between the following protons and carbons: $3-\mathrm{H}$ and the formyl ester carbonyl carbon $\left(\delta_{\mathrm{C}} 160.7\right) ; 23-\mathrm{H}_{3}$ and $3-5$, $24-\mathrm{C} ; 24-\mathrm{H}_{3}$ and $3-5,23-\mathrm{C} ; 25-\mathrm{H}_{3}$ and $1,5,9,10-\mathrm{C} ; 26-\mathrm{H}_{3}$ and $7-9,14-\mathrm{C} ; 27-\mathrm{H}_{3}$ and $8,13-15-\mathrm{C} ; 28-\mathrm{H}_{3}$ and $16-18$, 22-C; the formyl proton and 3-C. Finally, alkaline hydrolysis of 1 with $10 \%$ aqueous potassium hydroxide $(\mathrm{KOH})-50 \%$ aqueous 1,4-dioxane $(1: 1, \mathrm{v} / \mathrm{v})$ liberated 3-epi- $\alpha$-amyrin ${ }^{37)}$ (1a), so that the stereostructure of olibanumol $\mathrm{K}$ was determined to be 3-epi- $\alpha$-amyrin formate (1).

Structures of Olibanumols L (2), M (3), and N (4) Olibanumol L (2) was also obtained as a white powder with positive optical rotation $\left([\alpha]_{\mathrm{D}}^{28}+14.5\right.$ in $\left.\mathrm{MeOH}\right)$ and showed absorption bands at 3432 and $1739 \mathrm{~cm}^{-1}$ due to hydroxyl and 




Chart 2

Table 1. ${ }^{13} \mathrm{C}-\mathrm{NMR}$ Data for Olibanumols K (1), L (2), M (3), and N (4)

\begin{tabular}{|c|c|c|c|c|}
\hline Position & 1 & 2 & 3 & 4 \\
\hline 1 & 33.8 & 35.7 & 33.6 & 34.9 \\
\hline 2 & 23.0 & 22.8 & 25.5 & 22.9 \\
\hline 3 & 78.5 & 78.0 & 75.9 & 78.1 \\
\hline 4 & 36.5 & 36.5 & 37.5 & 36.5 \\
\hline 5 & 50.0 & 49.9 & 48.8 & 49.8 \\
\hline 6 & 18.2 & 18.1 & 18.3 & 18.1 \\
\hline 7 & 32.8 & 33.3 & 33.6 & 33.1 \\
\hline 8 & 40.2 & 43.4 & 43.5 & 43.0 \\
\hline 9 & 47.5 & 55.5 & 51.8 & 52.6 \\
\hline 10 & 36.9 & 38.0 & 38.1 & 38.0 \\
\hline 11 & 23.3 & 68.1 & 76.6 & 76.7 \\
\hline 12 & 124.3 & 128.4 & 124.0 & 123.5 \\
\hline 13 & 139.5 & 142.7 & 143.4 & 143.3 \\
\hline 14 & 42.2 & 42.1 & 42.1 & 42.1 \\
\hline 15 & 28.2 & 27.9 & 27.9 & 27.9 \\
\hline 16 & 26.7 & 26.7 & 26.7 & 26.8 \\
\hline 17 & 33.8 & 33.5 & 33.4 & 33.5 \\
\hline 18 & 59.1 & 58.0 & 58.5 & 58.4 \\
\hline 19 & 39.7 & 39.4 & 39.5 & 39.5 \\
\hline 20 & 39.7 & 39.3 & 39.3 & 39.3 \\
\hline 21 & 31.3 & 31.1 & 31.1 & 31.1 \\
\hline 22 & 41.6 & 41.2 & 41.4 & 41.4 \\
\hline 23 & 28.8 & 28.6 & 28.6 & 28.1 \\
\hline 24 & 22.0 & 22.0 & 22.4 & 22.0 \\
\hline 25 & 15.6 & 16.6 & 16.8 & 16.9 \\
\hline 26 & 17.0 & 18.1 & 18.2 & 18.2 \\
\hline 27 & 23.5 & 23.2 & 22.6 & 22.6 \\
\hline 28 & 28.1 & 28.7 & 28.7 & 28.7 \\
\hline 29 & 17.6 & 17.5 & 17.4 & 17.3 \\
\hline 30 & 21.5 & 21.3 & 21.3 & 21.4 \\
\hline HCOO- & 160.7 & & & \\
\hline $\mathrm{CH}_{3} \mathrm{CO}-$ & & 170.5 & & 170.6 \\
\hline $\mathrm{CH}_{3} \mathrm{CO}-$ & & 21.4 & & 21.3 \\
\hline $\mathrm{CH}_{3} \mathrm{O}-$ & & & 54.2 & 54.7 \\
\hline
\end{tabular}

Measured in $\mathrm{CDCl}_{3}$.

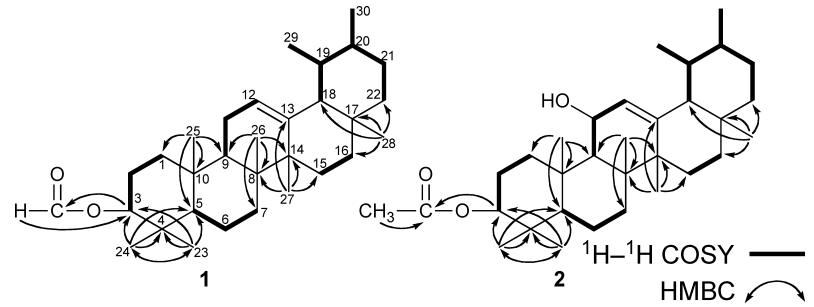

Fig. 1. ${ }^{1} \mathrm{H}-{ }^{1} \mathrm{H}$ COSY and $\mathrm{HMBC}$ Experiments of $\mathbf{1}$ and $\mathbf{2}$

ester carbonyl functions in the IR spectrum. The molecular formula $\mathrm{C}_{32} \mathrm{H}_{52} \mathrm{O}_{3}$ was determined from the positive-ion FAB-MS $\left[\mathrm{m} / \mathrm{z} 507(\mathrm{M}+\mathrm{Na})^{+}\right]$and by high-resolution MS measurement. The ${ }^{1} \mathrm{H}-\left(\mathrm{CDCl}_{3}\right)$ and ${ }^{13} \mathrm{C}-\mathrm{NMR}$ (Table 1) spectra of $\mathbf{2}$ indicated the presence of eight methyls $[\delta 0.81$, $0.86,0.93,1.19\left(3 \mathrm{H}\right.$ each, all s, 28, 24, 23, 27- $\left.\mathrm{H}_{3}\right), 1.11(6 \mathrm{H}$, s, 25, 26- $\left.\mathrm{H}_{3}\right), 0.86,0.88(3 \mathrm{H}$ each, both d, $J=7.6 \mathrm{~Hz}, 29,30-$ $\left.\left.\mathrm{H}_{3}\right)\right]$, an acetyl $\left[\delta 2.03\left(3 \mathrm{H}, \mathrm{s}, \mathrm{CH}_{3} \mathrm{CO}-\right)\right]$, two methines bearing an oxygen function $[\delta 4.27(1 \mathrm{H}$, dd-like, $11-\mathrm{H}), 4.61$ $(1 \mathrm{H}$, br s, $3-\mathrm{H})]$, and an olefin $[\delta 5.18(1 \mathrm{H}$, br s, $12-\mathrm{H})]$ together with eight methylenes, five methines, and seven quaternary carbons. As shown in Fig. 1 , the ${ }^{1} \mathrm{H}-{ }^{1} \mathrm{H}$ COSY experiment on $\mathbf{2}$ indicated the presence of partial structures written in bold lines. In the HMBC experiment on 2, long-range correlations were observed between the following protons and carbons: $3-\mathrm{H}$ and the acetyl carbonyl carbon $\left(\delta_{\mathrm{C}} 170.5\right) ; 23-$ $\mathrm{H}_{3}$ and $3-5,24-\mathrm{C} ; 24-\mathrm{H}_{3}$ and $3-5,23-\mathrm{C} ; 25-\mathrm{H}_{3}$ and $1,5,9$, $10-\mathrm{C} ; 26-\mathrm{H}_{3}$ and $7-9,14-\mathrm{C} ; 27-\mathrm{H}_{3}$ and $8,13-15-\mathrm{C} ; 28-\mathrm{H}_{3}$ and $16-18,22-\mathrm{C}$. Thus, the connectivities of quaternary carbons $(4,8,10,13,14,17-\mathrm{C})$ and position of the acetyl group in 2 were clarified and its urs-12-ene-3,11-diol structure was elucidated. Finally, deacetylation of 2 with $0.14 \%$ 
sodium methoxide $(\mathrm{NaOMe})-\mathrm{MeOH}$ yielded urs-12-ene$3 \alpha, 11 \alpha$-diol (17). Consequently, the stereostructure of olibanumol L was determined to be 3-O-acetyl-urs-12-ene$3 \alpha, 11 \alpha$-diol (2).

Olibanumol M (3), a white powder with positive optical rotation $\left([\alpha]_{\mathrm{D}}^{27}+21.0\right.$ in $\left.\mathrm{MeOH}\right)$, showed absorption bands at 3475 and $1735 \mathrm{~cm}^{-1}$ due to hydroxyl and olefin functions in the IR spectrum. The positive-ion FAB-MS spectrum of 3 showed a quasimolecular ion peak at $m / z 479(\mathrm{M}+\mathrm{Na})^{+}$, and the molecular formula was determined to be $\mathrm{C}_{31} \mathrm{H}_{52} \mathrm{O}_{2}$ by high-resolution MS measurement. The ${ }^{1} \mathrm{H}-\left(\mathrm{CDCl}_{3}\right)$ and ${ }^{13} \mathrm{C}-$ NMR (Table 1) spectra of $\mathbf{3}$ indicated the presence of an urs12-ene-3,11-diol part $[\delta 0.80,0.87,0.97,1.08,1.11,1.16$ (3H each, all s, 28, 24, 23, 25, 26, 27- $\left.\mathrm{H}_{3}\right), 0.86,0.88(3 \mathrm{H}$ each, both d, $\left.J=7.6 \mathrm{~Hz}, 29,30-\mathrm{H}_{3}\right), 3.39(1 \mathrm{H}, \mathrm{br} \mathrm{s}, 3-\mathrm{H}), 3.85$ $(1 \mathrm{H}, \mathrm{dd}, J=3.9,9.2 \mathrm{~Hz}, 11-\mathrm{H}), 5.33(1 \mathrm{H}, \mathrm{d}-\mathrm{like}, 12-\mathrm{H})]$ together with a methoxyl group $\left[\delta 3.26\left(3 \mathrm{H}, \mathrm{s}, \mathrm{CH}_{3} \mathrm{O}-\right)\right]$. On the other hand, olibanumol $\mathrm{N}(4), \mathrm{C}_{33} \mathrm{H}_{54} \mathrm{O}_{3}$, was also obtained as a white powder with positive optical rotation $\left([\alpha]_{D}^{22}+1.1\right.$ in $\left.\mathrm{MeOH}\right)$. The ${ }^{1} \mathrm{H}-\left(\mathrm{CDCl}_{3}\right)$ and ${ }^{13} \mathrm{C}-\mathrm{NMR}$ (Table 1) spectra of $\mathbf{4}$ were similar to those of $\mathbf{2}$, except for the signals due to the 11-methoxyl group $[\delta 3.28(3 \mathrm{H}, \mathrm{s}$, $\left.\left.\mathrm{CH}_{3} \mathrm{O}-\right)\right]$. Methylation of $\mathbf{2}$ with methyl iodide $\left(\mathrm{CH}_{3} \mathrm{I}\right)$ and potassium carbonate $\left(\mathrm{K}_{2} \mathrm{CO}_{3}\right)$ yielded 4 , whereas acetylation of 3 with $\mathrm{Ac}_{2} \mathrm{O}$ /pyridine furnished 4 . On the basis of this evidence, the stereostructures of olibanumols $\mathrm{M}$ and $\mathrm{N}$ were determined to be $11 \alpha$-methyurs-12-ene-3 $\alpha, 11 \alpha$-diol (3) and its acetate (4).

\section{Experimental}

The following instruments were used to obtain spectral and physical data: specific rotations, Horiba SEPA-300 digital polarimeter $(l=5 \mathrm{~cm})$; IR spectra, Shimadzu FTIR-8100 spectrometer; ${ }^{1} \mathrm{H}-\mathrm{NMR}$ spectra, JEOL JNMLA500 $(500 \mathrm{MHz})$ and EX-270 (270 MHz) spectrometers; ${ }^{13} \mathrm{C}-\mathrm{NMR}$ spectra, JEOL JNM-LA500 (125 MHz) and EX-270 $(68 \mathrm{MHz})$ spectrometers with tetramethylsilane as an internal standard; FAB-MS and high resolution FAB-MS, JEOL JMS-SX 102A mass spectrometer; HPLC detector, Shimadzu RID-6A refractive index and SPD-10A UV-VIS detectors; HPLC column, YMC-Pack ODS-A and YMC-Pack SIL (YMC Co., Ltd., Kyoto, Japan) $(250 \mathrm{~mm} \times 4.6 \mathrm{~mm}$ i.d. $)$ and $(250 \mathrm{~mm} \times 20 \mathrm{~mm}$ i.d. $)$ columns were used for analytical and preparative purposes, respectively.

The following experimental conditions were used for chromatography: normal-phase silica gel column chromatography (CC), silica gel 60N (Kanto Chemical Co., Ltd., 63-210 mesh, spherical, neutral); reversed-phase silica gel CC, Chromatorex ODS DM1020T (Fuji Silysia Chemical, Ltd., 100200 mesh); normal-phase TLC, pre-coated TLC plates with silica gel $60 \mathrm{~F}_{25}$ (Merck, $0.25 \mathrm{~mm}$ ); reversed-phase TLC, pre-coated TLC plates with silica gel RP-18 $\mathrm{F}_{254 \mathrm{~S}}$ (Merck, $0.25 \mathrm{~mm}$ ); reversed-phase HPTLC, pre-coated TLC plates with silica gel RP-18 $\mathrm{WF}_{254 \mathrm{~S}}$ (Merck, $0.25 \mathrm{~mm}$ ), detection was achieved by spraying with $1 \% \mathrm{Ce}\left(\mathrm{SO}_{4}\right)_{2}-10 \%$ aqueous $\mathrm{H}_{2} \mathrm{SO}_{4}$, followed by heating.

Plant Material This item was described in a previous report. ${ }^{10)}$

Extraction and Isolation The gum-resin from $B$. carterii $(2.7 \mathrm{~kg})$ was extracted three times with $80 \%$ aqueous acetone at room temperature for $24 \mathrm{~h}$. Evaporation of the solvent under reduced pressure provided an aqueous acetone extract $(2131 \mathrm{~g}, 78.9 \%)$. The aqueous acetone extract $(360.0 \mathrm{~g})$ was subjected to normal-phase silica gel CC $[3.0 \mathrm{~kg}, n$-hexane-EtOAc $(30$ $1 \rightarrow 20: 1 \rightarrow 10: 1 \rightarrow 1: 1, \mathrm{v} / \mathrm{v}) \rightarrow \mathrm{MeOH}$ ] to give 11 fractions [Fr. $1(6.3 \mathrm{~g}), \mathrm{Fr}$. 2 (11.2 g), Fr. 3 (30.5 g), Fr. 4 (181.4 g), Fr. 5 (12.7 g), Fr. 6 (9.3 g), Fr. 7 (18.2 g), Fr. 8 (14.6 g), Fr. 9 (7.1 g), Fr. 10 (5.9 g), and Fr. 11 (15.6 g)], as reported previously. ${ }^{10)}$ The fraction $2(11.2 \mathrm{~g})$ was subjected to normal-phase silica gel CC $[345 \mathrm{~g}, n$-hexane $\rightarrow n$-hexane-EtOAc $(100: 1 \rightarrow 80: 1 \rightarrow 30: 1 \rightarrow$ $20: 1 \rightarrow 10: 1, \mathrm{v} / \mathrm{v}) \rightarrow$ EtOAc] and HPLC [YMC-Pack SIL, UV detector $(254 \mathrm{~nm}), n$-hexane-EtOAc $(300: 1, \mathrm{v} / \mathrm{v})]$ to give olibanumol $\mathrm{K}(\mathbf{1}, 5.8 \mathrm{mg}$, $0.0013 \%)$ together with olibanumol $\mathrm{F}^{13)}(37.8 \mathrm{mg}, 0.0083 \%)$. The fraction 3 $(3.0 \mathrm{~g})$ was subjected to normal-phase silica gel CC [150 g, $n$-hexane-EtOAc $(30: 1, \mathrm{v} / \mathrm{v}) \rightarrow \mathrm{MeOH}]$ to give epilupeol acetate $(6,1288.9 \mathrm{mg}, 2.87 \%)$ and lupenone $(\mathbf{1 2}, 148.2 \mathrm{mg}, 0.33 \%)$. The fraction $5(12.7 \mathrm{~g})$ subjected by re- versed-phase silica gel $\mathrm{CC}\left[381 \mathrm{~g}, \mathrm{MeOH}-\mathrm{H}_{2} \mathrm{O}(70: 30 \rightarrow 80: 20 \rightarrow 90: 10\right.$, $\mathrm{v} / \mathrm{v}) \rightarrow \mathrm{MeOH}$ ] and HPLC [YMC-Pack ODS-A, RI detector, $\mathrm{MeOH}-\mathrm{H}_{2} \mathrm{O}$ $(90: 10, \mathrm{v} / \mathrm{v})]$ to furnish lupeol $(\mathbf{9}, 3289.2 \mathrm{mg}, 0.72 \%)$ together with olibanumol $\mathrm{D}^{13)}(22.8 \mathrm{mg}, 0.0050 \%)$. The fraction $6(3.0 \mathrm{~g})$ was subjected by reversed-phase silica gel $\mathrm{CC}\left[150 \mathrm{~g}, \mathrm{MeOH}-\mathrm{H}_{2} \mathrm{O}(70: 30 \rightarrow 80: 20 \rightarrow 90: 10\right.$, $\mathrm{v} / \mathrm{v}) \rightarrow \mathrm{MeOH}$ ] and HPLC [YMC-Pack ODS-A, RI detector, $\mathrm{MeOH}-\mathrm{H}_{2} \mathrm{O}$ $(90: 10$ or $95: 5, \mathrm{v} / \mathrm{v})]$ to give neoilexonol acetate $(15,338.2 \mathrm{mg}, 0.23 \%)$ and dammarenediol II acetate $(\mathbf{1 9}, 661.8 \mathrm{mg}, 0.45 \%)$. The fraction $7(9.0 \mathrm{~g})$ was subjected to normal-phase silica gel CC [270 g, $n$-hexane-EtOAc $(10: 1 \rightarrow$ $5: 1 \rightarrow 1: 1, \mathrm{v} / \mathrm{v}) \rightarrow \mathrm{MeOH}$ ] to give six fractions [Fr. 7-1 $(95.0 \mathrm{mg})$, Fr. 7-2 (369.7 mg), Fr. 7-3 (2.00 g), Fr. 7-4 (694.3 mg), Fr. 7-5 (3.50 g), and Fr. 7-6 $(2.10 \mathrm{~g})]$, as reported previously. ${ }^{13)}$ The fraction 7-3 (2.0 g) was purified by HPLC [YMC-Pack ODS-A, RI detector, $\mathrm{MeOH}-\mathrm{H}_{2} \mathrm{O}(95: 5, \mathrm{v} / \mathrm{v})$ ] to furnish $3 \beta$-acetoxylup-20(29)-en- $11 \beta$-ol (11,97.0 $\mathrm{mg}, 0.043 \%)$ and mansumbinol $(\mathbf{2 5}, 16.0 \mathrm{mg}, 0.0071 \%)$. The fraction 7-4 $(694.3 \mathrm{mg})$ was purified by HPLC [YMC-Pack ODS-A, RI detector, $\mathrm{MeOH}-\mathrm{H}_{2} \mathrm{O}(95: 5, \mathrm{v} / \mathrm{v})$ ] to furnish olibanumol L (2, $97.0 \mathrm{mg}, 0.043 \%)$ together with olibanumol $\mathrm{G}^{13)}(11.3 \mathrm{mg}$, $0.0050 \%)$. The fraction 7-5 (3.5 g) was subjected by reversed-phase silica gel $\mathrm{CC}\left[175 \mathrm{~g}, \mathrm{MeOH}-\mathrm{H}_{2} \mathrm{O}(70: 30 \rightarrow 80: 20 \rightarrow 90: 10, \mathrm{v} / \mathrm{v}) \rightarrow \mathrm{MeOH}\right]$ and HPLC [YMC-Pack ODS-A, RI detector, $\mathrm{MeOH}-\mathrm{H}_{2} \mathrm{O}(90: 10$ or $95: 5, \mathrm{v} / \mathrm{v})$ ] to give olibanumol N (4, $7.9 \mathrm{mg}, 0.0035 \%)$, glochidiol $(\mathbf{8}, 3.8 \mathrm{mg}, 0.0017 \%)$, neoilexonol (14, $88.0 \mathrm{mg}, 0.039 \%)$, and $15(97.0 \mathrm{mg}, 0.043 \%)$. The fraction 7-6 (2.1 g) was purified by HPLC [YMC-Pack ODS-A, RI detector, $\mathrm{MeOH}-1 \%$ aqueous $\mathrm{AcOH}(95: 5, \mathrm{v} / \mathrm{v})]$ to give dammarenodiol II (18, $200.8 \mathrm{mg}, 0.089 \%)$ and ocotillol acetate $(\mathbf{2 3}, 9.9 \mathrm{mg}, 0.0044 \%)$. The fraction $9(7.1 \mathrm{~g})$ subjected to reversed-phase silica gel CC $\left[200 \mathrm{~g}, \mathrm{MeOH}-\mathrm{H}_{2} \mathrm{O}\right.$ $(70: 30 \rightarrow 90: 10, \mathrm{v} / \mathrm{v}) \rightarrow \mathrm{MeOH}$ ] and HPLC [YMC-Pack ODS-A, RI detector, $\mathrm{MeOH}-\mathrm{H}_{2} \mathrm{O}(95: 5, \mathrm{v} / \mathrm{v})$ ] to give lup-20(30)-ene-3 $\alpha, 29$-diol (7, $22.8 \mathrm{mg}$, $0.0050 \%$ ), lup-20(29)-ene- $\alpha, 3 \beta$-diol (10, $11.8 \mathrm{mg}, 0.0026 \%)$, urs- $9(11)$, 12-dien-3 $\beta$-ol (13, $5.5 \mathrm{mg}, 0.0012 \%)$, urs-12-ene-3 $\beta, 11 \alpha$-diol (16, $18.7 \mathrm{mg}$, $0.0041 \%)$, urs-12-ene- $3 \alpha, 11 \alpha$-diol $(\mathbf{1 7}, 68.3 \mathrm{mg}, 0.015 \%)$, and 3 - $O$-acetyl$3 \beta, 20 S, 24$,trihydroxydammar-25-ene (20,10.0 $\mathrm{mg}, 0.0022 \%)$ together with olibanumol $\mathrm{E}^{13)}(13.2 \mathrm{mg}, 0.0029 \%)$. The fraction $10(5.9 \mathrm{~g})$ was subjected by reversed-phase silica gel CC $\left[180 \mathrm{~g}, \mathrm{MeOH}-\mathrm{H}_{2} \mathrm{O} \quad(70: 30 \rightarrow 80: 20 \rightarrow\right.$ $90: 10, \mathrm{v} / \mathrm{v}) \rightarrow \mathrm{MeOH}]$ and $\mathrm{HPLC}\left[\mathrm{MeOH}-\mathrm{H}_{2} \mathrm{O}(95: 5, \mathrm{v} / \mathrm{v})\right]$ to furnish olibanumol M (3, $19.7 \mathrm{mg}, 0.0043 \%)$, isofouquierol acetate $(\mathbf{2 2}, 301.9 \mathrm{mg}$, $0.066 \%)$ and $3 \beta$-hydroxymansumbin-13(17)-en-16-one $(\mathbf{2 4}, \quad 29.7 \mathrm{mg}$, $0.0065 \%)$

Olibanumol K (1): A white powder, $[\alpha]_{\mathrm{D}}^{27}+16.2(c=0.30, \mathrm{MeOH})$. Highresolution positive-ion FAB-MS: Calcd for $\mathrm{C}_{31} \mathrm{H}_{50} \mathrm{O}_{2} \mathrm{Na}(\mathrm{M}+\mathrm{Na})^{+}$ 477.3709; Found 477.3703. IR $\left(\mathrm{KBr}, \mathrm{cm}^{-1}\right)$ : 2940, 1725, 1457, 1379. ${ }^{1} \mathrm{H}-$ $\operatorname{NMR}\left(500 \mathrm{MHz}, \mathrm{CDCl}_{3}\right) \delta: 0.80,0.89,0.92,0.92,1.11,1.13$ (3H each, all s, $\left.28,23,24,25,27,26-\mathrm{H}_{3}\right), 0.90\left(3 \mathrm{H}, \mathrm{d}, J=7.4 \mathrm{~Hz}, 29-\mathrm{H}_{3}\right), 0.92(3 \mathrm{H}, \mathrm{d}, J=$ $\left.7.0 \mathrm{~Hz}, 30-\mathrm{H}_{3}\right), 4.77(3 \mathrm{H}$, br s, 3-H), $5.13(1 \mathrm{H}$, dd-like, $12-\mathrm{H}), 8.13(1 \mathrm{H}, \mathrm{s}$, $\mathrm{HCO}-) .{ }^{13} \mathrm{C}-\mathrm{NMR}\left(125 \mathrm{MHz}, \mathrm{CDCl}_{3}\right) \delta_{\mathrm{C}}$ : given in Table 1. Positive-ion FAB-MS $m / z$ : $477(\mathrm{M}+\mathrm{Na})^{+}$

Olibanumol L (2): A white powder, $[\alpha]_{\mathrm{D}}^{28}+14.5(c=1.00, \mathrm{MeOH})$. Highresolution positive-ion FAB-MS: Calcd for $\mathrm{C}_{32} \mathrm{H}_{52} \mathrm{O}_{3} \mathrm{Na}(\mathrm{M}+\mathrm{Na})^{+}$ 507.3805; Found 507.3814. IR $\left(\mathrm{KBr}, \mathrm{cm}^{-1}\right)$ : 3432, 2962, 1739, 1456, 1370. ${ }^{1} \mathrm{H}-\mathrm{NMR}\left(500 \mathrm{MHz}, \mathrm{CDCl}_{3}\right) \delta: 0.81,0.86,0.93,1.19$ (3H each, all s, 28, 24, $\left.23,27-\mathrm{H}_{3}\right), 1.11\left(6 \mathrm{H}, \mathrm{s}, 25,26-\mathrm{H}_{3}\right), 0.86,0.88(3 \mathrm{H}$ each, both d, $J=7.6 \mathrm{~Hz}$, $\left.29,30-\mathrm{H}_{3}\right), 2.03\left(3 \mathrm{H}, \mathrm{s}, \mathrm{CH}_{3} \mathrm{CO}-\right), 4.27(1 \mathrm{H}$, dd-like, 11-H), $4.61(1 \mathrm{H}, \mathrm{brs}$, $3-\mathrm{H}), 5.18(1 \mathrm{H}, \mathrm{br} \mathrm{s}, 12-\mathrm{H}) .{ }^{13} \mathrm{C}-\mathrm{NMR}\left(125 \mathrm{MHz}, \mathrm{CDCl}_{3}\right) \delta_{\mathrm{C}}$ : given in Table 1. Positive-ion FAB-MS m/z: $507(\mathrm{M}+\mathrm{Na})^{+}$.

Olibanumol M (3): A white powder, $[\alpha]_{\mathrm{D}}^{27}+21.0(c=1.00, \mathrm{MeOH})$. Highresolution positive-ion FAB-MS: Calcd for $\mathrm{C}_{31} \mathrm{H}_{52} \mathrm{O}_{2} \mathrm{Na}(\mathrm{M}+\mathrm{Na})^{+}$ 479.3814; Found 479.3805. IR ( $\left.\mathrm{KBr}, \mathrm{cm}^{-1}\right)$ : 3475, 2926, 1735, 1456, 1389. ${ }^{1} \mathrm{H}-\mathrm{NMR}\left(500 \mathrm{MHz}, \mathrm{CDCl}_{3}\right) \delta: 0.80,0.87,0.97,1.08,1.11,1.16(3 \mathrm{H}$ each, all s, 28, 24, 23, 25, 26, 27- $\left.\mathrm{H}_{3}\right), 0.86,0.88$ (3H each, both d, $J=7.6 \mathrm{~Hz}, 29$, $\left.30-\mathrm{H}_{3}\right), 3.26\left(3 \mathrm{H}, \mathrm{s}, \mathrm{CH}_{3} \mathrm{O}-\right), 3.39(1 \mathrm{H}$, br s, $3-\mathrm{H}), 3.85(1 \mathrm{H}, \mathrm{dd}, J=3.9$, $9.2 \mathrm{~Hz}, 11-\mathrm{H}), 5.33(1 \mathrm{H}$, d-like, $12-\mathrm{H}) .{ }^{13} \mathrm{C}-\mathrm{NMR}\left(125 \mathrm{MHz}, \mathrm{CDCl}_{3}\right) \delta_{\mathrm{C}}$ : given in Table 1. Positive-ion FAB-MS $m / z: 479(\mathrm{M}+\mathrm{Na})^{+}$

Olibanumol N (4): A white powder, $[\alpha]_{\mathrm{D}}^{22}+1.1(c=1.00, \mathrm{MeOH})$. Highresolution positive-ion FAB-MS: Calcd for $\mathrm{C}_{33} \mathrm{H}_{54} \mathrm{O}_{3} \mathrm{Na}(\mathrm{M}+\mathrm{Na})^{+} 521.3814$; Found 521.3805. IR $\left(\mathrm{KBr}, \mathrm{cm}^{-1}\right)$ : 2926, 1732, 1458, 1374. ${ }^{1} \mathrm{H}-\mathrm{NMR}(500$ $\left.\mathrm{MHz}, \mathrm{CDCl}_{3}\right) \delta: 0.81,0.86,0.93,1.04,1.08,1.19$ (3H each, all s, 28, 24, 23, $\left.25,26,27-\mathrm{H}_{3}\right), 0.86,0.88\left(3 \mathrm{H}\right.$ each, both d, $\left.J=7.6 \mathrm{~Hz}, 29,30-\mathrm{H}_{3}\right), 2.03(3 \mathrm{H}$, $\left.\mathrm{s}, \mathrm{CH}_{3} \mathrm{CO}-\right), 3.28\left(3 \mathrm{H}, \mathrm{s}, \mathrm{CH}_{3} \mathrm{O}-\right), 3.77(1 \mathrm{H}$, dd-like, 11-H), $4.61(1 \mathrm{H}, \mathrm{br}$, 3-H), $5.39(1 \mathrm{H}, \mathrm{d}-\mathrm{like}, 12-\mathrm{H}) .{ }^{13} \mathrm{C}-\mathrm{NMR}\left(125 \mathrm{MHz}, \mathrm{CDCl}_{3}\right) \delta_{\mathrm{C}}$ : given in Table 1. Positive-ion FAB-MS $m / z: 521(\mathrm{M}+\mathrm{Na})^{+}$.

Deformylation of 1 A solution of $1(3.0 \mathrm{mg})$ in $10 \%$ aqueous potassium hydroxide (KOH)-50\% aqueous 1,4-dioxane $(1: 1, \mathrm{v} / \mathrm{v}, 2.0 \mathrm{ml})$ was stirred at $37^{\circ} \mathrm{C}$ for $1 \mathrm{~h}$. The reaction mixture was neutralized with Dowex HCR W2 $\left(\mathrm{H}^{+}\right.$form $)$, and the resins were removed by filtration. After removal of the 
solvent under reduced pressure, the residue was separated by normal-phase silica gel CC $[1.0 \mathrm{~g}, n$-hexane-EtOAc $(3: 1, \mathrm{v} / \mathrm{v})]$ to give 3 -epi- $\alpha$-amyrin (1a, $0.8 \mathrm{mg}$ ), which was identified by comparison of its physical and spectral data $\left([\alpha]_{\mathrm{D}},{ }^{1} \mathrm{H}-\mathrm{NMR}\right.$, and MS) with reported values. ${ }^{37)}$

Deacetylation of 2 A solution of $2(10.0 \mathrm{mg})$ in $0.14 \%$ sodium methoxide $(\mathrm{NaOMe})-\mathrm{MeOH}(2.0 \mathrm{ml})$ was stirred at room temperature for $3 \mathrm{~h}$. The reaction mixture was neutralized with Dowex $\mathrm{HCR}$ W2 $\left(\mathrm{H}^{+}\right.$form $)$, and the resins were removed by filtration. After removal of the solvent under reduced pressure, the residue was separated by normal-phase silica gel CC $[1.0 \mathrm{~g}, n$-hexane-EtOAc $(3: 1, \mathrm{v} / \mathrm{v})]$ to give urs-12-ene-3 $\alpha, 11 \alpha$-diol (17, $7.5 \mathrm{mg}$ ), which was identified by comparison of its physical and spectral data $\left([\alpha]_{\mathrm{D}},{ }^{1} \mathrm{H}-\mathrm{NMR}\right.$, and MS) with reported values. ${ }^{28)}$

Methylation of 2 To a solution of $\mathbf{2}(10.0 \mathrm{mg})$ in dry dimethylformamide (DMF, $2.0 \mathrm{ml})$ was added methyl iodide $\left(\mathrm{CH}_{3} \mathrm{I}, 15 \mu \mathrm{l}\right)$ and potassium carbonate $\left(\mathrm{K}_{2} \mathrm{CO}_{3}, 66.4 \mathrm{mg}\right)$, and the mixture was stirred at $80^{\circ} \mathrm{C}$ for $3 \mathrm{~h}$. The reaction mixture was poured into brine and extracted with EtOAc. The extract was successively washed with saturated aqueous $\mathrm{NaHCO}_{3}$ and brine, and filtrated. After removal of the solvent under reduced pressure, the residue was purified by normal-phase silica gel CC [1.0 g, $n$-hexane-EtOAc $(3: 1, v / v)$ ] to give $4(7.0 \mathrm{mg})$.

Acetylation of 3 To a solution of $\mathbf{3}(5.0 \mathrm{mg})$ in dry pyridine $(2.0 \mathrm{ml})$ was added acetic anhydride $(1.0 \mathrm{ml})$, and the mixture was stirred at room temperature for $3 \mathrm{~h}$. The reaction mixture was poured into ice-water and extracted with EtOAc. The extract was successively washed with $5 \%$ aqueous $\mathrm{HCl}$, saturated aqueous $\mathrm{NaHCO}_{3}$, and brine, and filtrated. After removal of the solvent under reduced pressure, the residue was purified by normal-phase silica gel CC $[1.0 \mathrm{~g}, n$-hexane-EtOAc $(2: 1, \mathrm{v} / \mathrm{v})]$ to give $4(3.0 \mathrm{mg})$.

Acknowledgements M. Yoshikawa and H. Matsuda were supported by the 21st COE Program, Academic Frontier Project, and a Grant-in Aid for Scientific Research from MEXT (the Ministry of Education, Culture, Sports, Science and Technology of Japan). T. Morikawa was supported by 'HighTech Research Center' Project (2007-2011) and a Grant-in Aid for Scientific Research from MEXT. We also thank Mr. Nobuyuki Izumi for his personal financial support.

\section{References and Notes}

1) Morikawa T., Xu F., Matsuda H., Yoshikawa M., Heterocycles, 57, 1983-1988 (2002).

2) Yoshikawa M., Xu F., Morikawa T., Ninomiya K., Matsuda H., Bioorg. Med. Chem. Lett., 13, 1045-1049 (2003).

3) Yoshikawa M., Morikawa T., Xu F., Ando S., Matsuda H., Heterocycles, 60, 1787-1792 (2003).

4) Morikawa T., Xu F., Kashima Y., Matsuda H., Ninomiya K., Yoshikawa M., Org. Lett., 6, 869-872 (2004).

5) Morikawa T., Xu F., Ninomiya K., Matsuda H., Yoshikawa M., Chem. Pharm. Bull., 52, 494-497 (2004).

6) Xu F., Morikawa T., Matsuda H., Ninomiya K., Yoshikawa M., J. Nat. Prod., 67, 569-576 (2004).

7) Abdel-Halim O. B., Morikawa T., Ando S., Matsuda H., Yoshikawa M., J. Nat. Prod., 67, 1119-1124 (2004).

8) Morikawa T., Abdel Halim O. B., Matsuda H., Ando S., Muraoka O., Yoshikawa M., Tetrehedron, 62, 6435-6442 (2006).

9) Yoshikawa M., Morikawa T., Kobayashi H., Nakamura A., Matsuhira K., Nakamura S., Matsuda H., Chem. Pharm. Bull., 55, 428-434
(2007).

10) Yoshikawa M., Morikawa T., Oominami H., Matsuda H., Chem. Pharm. Bull., 57, 957-964 (2009).

11) Matsuda H., Nakashima S., Abdel-Halim O. B., Morikawa T., Yoshikawa M., Chem. Pharm. Bull., 58, 747-751 (2010).

12) Nakashima S., Matsuda H., Kurume A., Oda Y., Nakamura S., Yamashita M., Yoshikawa M., Bioorg. Med. Chem. Lett., 20, 2994-2997 (2010).

13) Morikawa M., Oominami H., Matsuda H., Yoshikawa M., J. Nat. Med., in press (2010).

14) Morikawa T., Matsuda H., Oominami H., Kageura T., Toguchida I., Yoshikawa M., "Abstract of Papers, the 43rd Symposium on the Chemistry of Natural Products," Osaka, October, 2001, pp. 485-490.

15) Ekong D. E. U., Okogun J. I., Phytochemistry, 8, 669-671 (1969).

16) Ullah N., Ahmed Z., Ahmed S., Muhammad P., Malik A., Phytochemistry, 50, 839-841 (1999).

17) Hui W.-H., Li M.-M., Phytochemistry, 15, 561-562 (1976).

18) Sholichin M., Yamasaki K., Kasai R., Tanaka O., Chem. Pharm. Bull., 28, 1006-1008 (1980).

19) Ageta H., Ageta T., Chem. Pharm. Bull., 32, 369-372 (1984).

20) Ghisalberti E. L., Jefferies P. R., Sefton M. A., Phytochemsitry, 12, $1125-1129$ (1973)

21) González A. G., Fraga B. M., González P., Marta M., Monache F. D., Marini-Bettolo G. B., De Mello J. F., Goncalves O., Phytochemistry, 21, 470- 471 (1982).

22) Hisham A., Kumar G. J., Fujimoto Y., Hara N., Phytochemsitry, 40, 1227-1231 (1995)

23) Lai J., Ito K., Chem. Pharm. Bull., 27, 2248-2251 (1979).

24) Matsunaga S., Tanaka R., Akagi M., Phytochemistry, 27, 535-537 (1988).

25) Yagishita K., Nishimura M., Agric. Biol. Chem., 25, 517-518 (1961).

26) Finucane B. W., Thomson J. B., J. Chem. Soc. Perkin 1, 1972, 18561862 (1972).

27) Ito K., Lai J., Yakugaku Zasshi, 98, 249-256 (1978).

28) De La Torre M. C., Bruno M., Piozzi F., Savona G., Rodriguez B., Arnold N. A., Phytochemstry, 29, 668-670 (1990).

29) Iwamoto M., Fujioka T., Okabe H., Mihashi K., Yamauchi T., Chem. Pharm. Bull., 35, 553-561 (1987).

30) Bianchini J.-P., Gaydou E. M., Rafaralahitsimba G., Waegell B., Zahra J.-P., Phytochemistry, 27, 2301-2304 (1988).

31) Butruille D., Dominguez X. A., Tetrahedron Lett., 8, 639-642 (1974).

32) Biftu T., Stevenson R., J. Chem. Soc. Perkin 1, 1978, 360-363 (1978).

33) Ohmoto T., Nikaido T., Ikuse M., Chem. Pharm. Bull., 26, 14371442 (1978).

34) Provan G. J., Gray A. I., Waterman P. G., Phytochemistry, 31, 20652068 (1992).

35) Provan G. J., Waterman P. G., Phytochemistry, 25, 917—922 (1986).

36) The ${ }^{1} \mathrm{H}$ - and ${ }^{13} \mathrm{C}$-NMR spectra of $\mathbf{1}-\mathbf{4}$ were assigned with the aid of distortionless enhancement by polarization transfer (DEPT), double quantum filter correlation spectroscopy (DQF COSY), heteronuclear multiple quantum coherence (HMQC), and heteronuclear multiple bond connectivity (HMBC) experiments.

37) Tamai M., Watanabe N., Someya M., Kondoh H., Omura S., Ling Z. P., Chang R., Ming C. W., Planta Med., 55, 44- 47 (1989). 